\title{
L1-based prototypicality effects in L2 vocabulary learning
}

\author{
Xia, Xiaoyan; Leung, Janny \\ Beijing Normal University
}

\begin{abstract}
Prototypicality effects in L1 vocabulary learning are well-documented (e.g., Rosch, 1973; Meints, Plunkett, \& Harris, 1999). Words for prototypical instances of a conceptual category are more quickly encoded into memory and more readily retrieved than words for nonprototypical instances. However, it remains unclear whether and how the effects will be present in the L2 context, the study of which could potentially illuminate the debate on unitary versus dual conceptual models in bilingualism. For instance, will the prototypicality effects be L1-based or L2-based, if cultural variation in prototypicality exists? What are the moderating factors?
\end{abstract}

A combination of qualitative and quantitative approaches is adopted to address the above questions. The conceptual categories under investigation were polysemous words, namely lexical items (e.g., red) with multiple distinct but related senses (e.g., prosperous, vicious, etc). The qualitative data, collected by questionnaires which were composed of a free senselisting task and a prototypicality rating task, were provided by 73 Chinese-speaking college students and 51 English-speaking American college students. These data were used to locate cultural variations in the prototypicality of the collected senses of a list of polysemous words. The quantitative data were obtained by an empirical experiment of a no-cued English-wordlearning and immediate-cued recall task, with 46 Chinese-speaking college freshmen as participants. The conceptual categories concerned were senses of white, yellow, dog, bamboo, dragon, green, and red. In the word-learning section, participants were instructed to learn by themselves pairs of novel English adjectives which designated category instances (i.e., word senses) of either L1p-L2np, e.g., prosperous, or L2p-L1np, e.g., vicious. But those English adjectives were not cued by their respective lexical items. For instance, prosperous was not cued by 红 (hong, Chinese character for red) and vicious not by red. In the cued-recall section, participants were told to group the previously-learned English adjectives (e.g., prosperous, vicious) according to the given cues (e.g., red or 红). The hypothesis proposed was that L2 words designating the L1p-L2np instances were retrieved better in terms of quantity and faster in terms of production order than those designating the L2p-L1np instances. Regarding production quantity, related-Sample $\mathrm{T}$ tests were used to compare the means of the recalled L1p-L2np and L2p-L1np instances. Regarding production order, frequency statistics and Chi-Square tests were used to test whether L1p-L2np instances were retrieved prior to L2p-L1np instances in participants' first responses to the given cues.

Our findings confirmed the hypothesis that L1-based prototypicality effects are present in L2 vocabulary learning. We argue that the effect of the L1-based, prototypical conceptual salience in L2 vocabulary learning is not static but dynamic, being influenced by factors such 
as formal instruction and exposure to the L2 and its culture. Possible means to reduce the L1based prototypicality effects are discussed.

\section{References:}

Rosch, E. H. (1973). Natural categories. Cognitive Psychology, 4(3), 328-350.

Meints, K., Plunkett, K., \& Harris, P. L. (1999). When does an ostrich become a bird? The role of typicality in early word comprehension. Developmental Psychology, 35(4), 1072-1078. 